\title{
Unhealthy Phenotype as Indicated by Salivary Biomarkers: Glucose, Insulin, VEGF-A, and IL-12p70 in Obese Kuwaiti Adolescents
}

\author{
Mor-Li Hartman, ${ }^{1}$ J. Max Goodson, ${ }^{1}$ Ping Shi, ${ }^{1}$ Jorel Vargas, ${ }^{1}$ \\ Tina Yaskell, ${ }^{1}$ Danielle Stephens, ${ }^{1}$ Maryann Cugini, ${ }^{1}$ Hatice Hasturk, \\ Roula Barake, ${ }^{2}$ Osama Alsmadi, ${ }^{3}$ Sabiha Al-Mutawa, ${ }^{4}$ Jitendra Ariga, \\ Pramod Soparkar, ${ }^{1}$ Jawad Behbehani, ${ }^{5}$ Kazem Behbehani, ${ }^{6}$ and Francine Welty ${ }^{7}$ \\ ${ }^{1}$ Department of Applied Oral Sciences, The Forsyth Institute, Cambridge, MA 02142, USA \\ ${ }^{2}$ Department of Nutrition, Dasman Diabetes Institute, 15462 Dasman, Kuwait \\ ${ }^{3}$ Genome Center, The Dasman Diabetes Institute, 15462 Dasman, Kuwait \\ ${ }^{4}$ Ministry of Health, 13001 Safat, Kuwait \\ ${ }^{5}$ Faculty of Dentistry, Kuwait University, 13060 Safat, Kuwait \\ ${ }^{6}$ Dasman Diabetes Institute, 15462 Dasman, Kuwait \\ ${ }^{7}$ Division of Cardiology, Beth Israel Deaconess Medical Center, Boston, MA 02215, USA
}

Correspondence should be addressed to Mor-Li Hartman; mhartman@forsyth.org

Received 25 November 2015; Accepted 11 February 2016

Academic Editor: Aron Weller

Copyright (c) 2016 Mor-Li Hartman et al. This is an open access article distributed under the Creative Commons Attribution License, which permits unrestricted use, distribution, and reproduction in any medium, provided the original work is properly cited.

\begin{abstract}
Objective. Here, we investigated the relationships between obesity and the salivary concentrations of insulin, glucose, and 20 metabolic biomarkers in Kuwaiti adolescents. Previously, we have shown that certain salivary metabolic markers can act as surrogates for blood concentrations. Methods. Salivary samples of whole saliva were collected from 8,317 adolescents. Salivary glucose concentration was measured by a high-sensitivity glucose oxidase method implemented on a robotic chemical analyzer. The concentration of salivary insulin and 20 other metabolic biomarkers was assayed in 744 randomly selected saliva samples by multiplexed bead-based immunoassay. Results. Obesity was seen in $26.5 \%$ of the adolescents. Salivary insulin predicting hyperinsulinemia occurred in $4.3 \%$ of normal-weight adolescents, $8.3 \%$ of overweight adolescents, and $25.7 \%$ of obese adolescents $(p<0.0001)$. Salivary glucose predicting hyperglycemia was found in only $3 \%$ of obese children and was not predictive $(p=0.89)$. Elevated salivary glucose and insulin occurring together was associated with elevated vascular endothelial growth factor and reduced salivary interleukin-12. Conclusion. Considering the surrogate nature of salivary insulin and glucose, this study suggests that elevated insulin may be a dominant sign of metabolic disease in adolescent populations. It also appears that a proangiogenic environment may accompany elevated glucose in obese adolescents.
\end{abstract}

\section{Introduction}

Overweight and obesity among children and adolescents is a global health problem, affecting not only developed nations but also low- to middle-income countries [1]. Childhood obesity is an independent risk factor for adult obesity [2] and has been linked to significant medical, social, academic, and psychological consequences [3]. In all age ranges, obesity is strongly associated with adverse health effects including hypertension, dyslipidemia, insulin resistance, hyperglycemia, and type 2 diabetes [4]. Given these data, it is of critical importance to identify obese children and adolescents with unfavorable metabolic risk factors who are at increased risk of developing comorbidities associated with obesity. Early detection may allow for early intervention, potentially preventing the development of chronic diseases. 
Traditionally, studies to identify adverse metabolic risk factors in children have focused on the levels of glucose, insulin, lipids, and/or biomarkers in serum or plasma. However, blood sampling can be particularly difficult for metabolic disease studies involving large cohorts of children, for whom the frequency of adverse reactions related to obtaining blood samples becomes an important limiting factor [5]. In one metabolic study of 12,761 children aged 11 to 13 years, a total of 256 adverse events (2\%) that were related to obtaining intravenous blood samples were reported [6]. Most were mild, but 35 instances of loss of consciousness were reported $(0.3 \%)$. Because of these adverse events, on-site emergency medical personnel were maintained during blood drawing as a precaution. In many types of studies, nonfatal adverse events occurring at a frequency of $2 \%$ would be considered acceptable; however, parental consent is a major limiting factor in enrollment for large metabolic studies from which the children derive no direct benefit. Therefore, if blood sampling studies are the only option, many unanswered questions in the field of obesity research will likely remain [5].

The objective of our investigation is to study the development of metabolic diseases in children. To investigate adverse metabolic marker progression in adolescents across body weight categories, we have been conducting studies based on saliva sample analysis rather than on blood sampling. This allows for studies of sufficient size to be performed with a high degree of child acceptance. We have been able to noninvasively collect data from over 8,000 10- to 11-year-old adolescents [7] who live in Kuwait, a country where over half of the adult population is obese [8] and one-quarter of adults have type 2 diabetes [9]. We recently published evidence which confirmed that salivary glucose concentration is significantly correlated with blood glucose concentration in 10year-old adolescents [10]. The relationship is defined as [plasma concentration $=13.5 \times$ saliva concentration + 84.8 ], in which $84.8 \mathrm{mg} / \mathrm{dL}$ is a salivary glucose threshold concentration. In that analysis, we found that a salivary glucose concentration $\geq 1.13 \mathrm{mg} / \mathrm{dL}$ was equivalent to the hyperglycemic blood glucose concentration $\geq 100 \mathrm{mg} / \mathrm{dL}$. In a second study, we analyzed the concentration of several protein biomarkers in saliva samples from a subset of 744 adolescents selected randomly from the larger study population and found that obese adolescents had significantly elevated levels of insulin, C-reactive protein, and leptin, along with significantly reduced levels of adiponectin, when compared to their normal-weight counterparts [7]. In the current report, we have expanded our previous studies by using high-throughput methodology to analyze metabolic markers in 8,245 saliva samples given by the Kuwaiti adolescents across every body weight category. Using this data, we evaluated the relationship between salivary insulin and glucose concentrations and obesity and identified various clinical and biomarker predictors of elevated salivary glucose concentration by body weight category.

\section{Methods}

2.1. Study Population and Design. The Kuwait study population and study design have been previously described [7]. Adolescents enrolled were native Kuwaitis who were in 4 th or 5th grade and were approximately equally distributed among each region (governorate) of Kuwait. A total of 8,317 adolescents participated in the study during 182 visits to 138 Kuwaiti schools between October 2, 2011, and May 15, 2012. The study was approved by the Dasman Diabetes Institute Ethical Review Committee in Kuwait. Arabic language informed consent was signed by parents/guardians in advance. Participant assent was obtained from the adolescents on the day of their evaluation.

\subsection{Salivary Glucose Concentration Analysis. Approximately} $3 \mathrm{~mL}$ of saliva was collected from each child in a $\geq 6$-hour fasting state, as described previously [7]. The mean salivary flow rate was $26 \pm 15.6 \mathrm{~mL} /$ hour $(0.43 \mathrm{~mL} / \mathrm{min})$, such that, on average, a $3 \mathrm{~mL}$ sample was collected in 6.9 minutes. Saliva samples were transported on ice from the collection site to the Tissue Bank Core Facility in the Dasman Institute for storage and analysis.

All saliva samples were weighed to determine their volume and centrifuged at $2,800 \mathrm{RPM}$ at $4^{\circ} \mathrm{C}$ for $20 \mathrm{~min}$ utes to remove particulate debris and exfoliated mucosal cells. For salivary glucose determination, $400 \mu \mathrm{L}$ of the supernatant was transferred to a screw cap 2D barcoded storage tube (Matrix ${ }^{\mathrm{TM}}$ tube, Thermo Fisher Scientific Inc., Waltham, Massachusetts, USA), and the tube was read by a barcode reader (Thermo Scientific VisionMate ST, Thermo Fisher Scientific Inc., Waltham, Massachusetts, USA). During sample preparation, the barcode was captured with subject number to a spreadsheet. The tubes were sealed by a torque-controlled tube capper (Thermo Scientific 8Channel Screw Cap Tube Capper, Thermo Fisher Scientific Inc., Waltham, Massachusetts, USA) and placed in a 96vial rack (Thermo Scientific Latch Rack, Thermo Fisher Scientific Inc., Waltham, Massachusetts, USA). Because the glucose assay used a high-sensitivity fluorescent method involving a peroxide intermediate, the capped 96-vial rack was boiled for 1 hour without loss of volume to denature the peroxidase and catalase known to be endogenously present in saliva [11-13]. After boiling, sample racks were maintained frozen at $-80^{\circ} \mathrm{C}$. Samples were air-transferred from Kuwait under temperature-monitored dry ice (Biocair, Boston, Massachusetts, USA) to the Forsyth Institute and were maintained at $-80^{\circ} \mathrm{C}$ until assay (average time to assay $=$ $0.88 \pm 0.06 \mathrm{y})$.

The measurement of salivary glucose was by fluorescent assay (Glucose Colorimetric/Fluorometric Assay Kit \#K606100, BioVision, Inc., Mountain View, CA, USA) implemented on a Tecan Freedom EVO ${ }^{\circledR} 150$ robotic processor with an 8channel liquid handling arm (Tecan Group Ltd., Männedorf, Switzerland). By this assay, an enzyme mixture specifically oxidizes glucose to generate hydrogen peroxide, which then reacts with a dye to generate color $(\lambda=570 \mathrm{~nm})$ and fluorescence $(\mathrm{Ex} / \mathrm{Em}=535 / 587 \mathrm{~nm})$. The fluorescence was measured by a spectrophotometer (Infinite ${ }^{\circledR} 200$ Pro, Tecan 
TABLE 1: Demographic data and clinical characteristics of 8,317 enrolled children overall and by weight category.

\begin{tabular}{|c|c|c|c|c|c|}
\hline & \multicolumn{4}{|c|}{ Body weight category } & \multirow{2}{*}{ Overall } \\
\hline & Underweight & Normal weight & Overweight & Obese & \\
\hline$N(\%)$ & $193(2.3)$ & $4,129(49.6)$ & $1,791(21.5)$ & $2,204(26.5)$ & $8,317(100)$ \\
\hline Age $(y)^{a}$ & $9.8 \pm 0.6$ & $9.9 \pm 0.7$ & $10.0 \pm 0.7$ & $10.1 \pm 0.7$ & $10.0 \pm 0.7$ \\
\hline Waist circumference $(\mathrm{cm})^{\mathrm{b}}$ & $54.3 \pm 5.1$ & $60.4 \pm 10.1$ & $70.2 \pm 7.1$ & $84.1 \pm 28.3$ & $68.7 \pm 19.4$ \\
\hline BMI $\left(\mathrm{kg} / \mathrm{m}^{2}\right)^{\mathrm{b}}$ & $13.5 \pm 0.8$ & $17.1 \pm 1.6$ & $21.7 \pm 1.3$ & $27.9 \pm 4.3$ & $20.9 \pm 5.3$ \\
\hline Salivary glucose concentration $(\mathrm{mg} / \mathrm{dL})$ & $0.20 \pm 0.25$ & $0.20 \pm 0.25$ & $0.18 \pm 0.22$ & $0.20 \pm 0.26$ & $0.19 \pm 0.24$ \\
\hline Salivary glucose $\geq 1.13 \mathrm{mg} / \mathrm{dL}, n(\%)$ & $4(2.1)$ & $72(1.7)$ & $27(1.5)$ & $38(1.7)$ & $141(1.7)$ \\
\hline Saliva flow rate $(\mathrm{mL} / \mathrm{h})^{\mathrm{c}}$ & $22.8 \pm 12.4$ & $24.9 \pm 14.5$ & $26.8 \pm 15.7$ & $27.8 \pm 17.2$ & $26.0 \pm 15.6$ \\
\hline Diastolic blood pressure $(\mathrm{mm} \mathrm{Hg})^{a}$ & $69.2 \pm 12.4$ & $70.2 \pm 16.2$ & $75.5 \pm 19.3$ & $81.3 \pm 27.0$ & $74.3 \pm 20.7$ \\
\hline Systolic blood pressure $(\mathrm{mm} \mathrm{Hg})^{\mathrm{a}}$ & $101.1 \pm 13.9$ & $103.3 \pm 14.8$ & $112.3 \pm 14.4$ & $119.4 \pm 14.8$ & $109.4 \pm 16.3$ \\
\hline Hypertension, $N(\%)^{\mathrm{a}}$ & $26(13.5)$ & $574(13.9)$ & $426(23.8)$ & $964(43.8)$ & $1990(23.9)$ \\
\hline
\end{tabular}

aAll significantly different except for "underweight" and "normal weight" $(p<0.001)$.

${ }^{\mathrm{b}}$ All categories significantly different $(p<0.001)$.

cAll categories significantly different except for "overweight" compared to "obese" $(p<0.001)$.

Group Ltd., Männedorf, Switzerland) using reverse 96-well plate reading mode. The 3-sigma detection limit of the glucose assay was $0.002 \mathrm{mg} / \mathrm{dL}$. Thirty microliters of saliva supernatant was assayed for each sample. Standards of 0.12 , $0.24,0.48$, and $0.96 \mathrm{mg} / \mathrm{dL}$ were assayed in triplicate on each run. Upon testing, samples that had been stored at $-80^{\circ} \mathrm{C}$ for varying periods of time up to one year were found to undergo a slow but significant reduction in glucose concentration (Figure S1 in Supplementary Material available online at http://dx.doi.org/10.1155/2016/6860240) at a rate of $0.242 \mathrm{mg} / \mathrm{dL} /$ year $\left(r^{2}=0.55, p<0.0001\right)$. Since the assay time and collection time of each of our samples were recorded, we were able to accurately compute the elapsed time and correct for this temporal degradation by adding the computed value to measured values. Corrected values are used in this report.

2.3. Analysis of Salivary Biomarkers. The salivary concentration of 20 biomarkers was evaluated using multiplex assays on a random cohort of 744 saliva samples taken from the larger population of 8,245 saliva samples, as described previously [7]. The 20 biomarkers included insulin, interferon- $\gamma$ (IFN$\gamma$ ), interleukin-10 (IL-10), IL-12p70, IL-13, IL-17A, IL-1 $\beta$, IL-4, IL-6, IL-8, monocyte chemotactic protein-1 (MCP1 ), tumor necrosis factor- $\alpha$ (TNF- $\alpha$ ), vascular endothelial growth factor-A (VEGF-A), ghrelin, leptin, myeloperoxidase, matrix metalloproteinase-9 (MMP-9), adiponectin (total), Creactive protein $(\mathrm{CRP})$, and resistin.

2.4. Statistical and Analytical. Body weight categories used in the analyses were those defined by the World Health Organization (WHO) using a body mass index (BMI) Zscore [14]. By this criterion, "obese" was $\geq 95$ th percentile, "overweight" was $\geq 85$ th to $<95$ th percentile, "normal weight" was $\geq 5$ th to $<85$ th percentile, and "underweight" was $<5$ th percentile. Hypertension was defined as $\geq 130 \mathrm{~mm} \mathrm{Hg}$ systolic or $\geq 85 \mathrm{~mm} \mathrm{Hg}$ diastolic blood pressure [15]. High salivary insulin was defined as $>177 \mathrm{pg} / \mathrm{mL}$ (equivalent to approximately $15 \mu \mathrm{U} / \mathrm{mL}$ in blood) [16]. High salivary glucose was defined as $\geq 1.13 \mathrm{mg} / \mathrm{dL}$ (equivalent to approximately $\geq 100 \mathrm{mg} / \mathrm{dL}$ in blood) [10].

To evaluate the relationship between salivary glucose concentration and salivary biomarker concentrations, we used stepwise selection to develop a parsimonious linear model to predict saliva glucose level (SAS 9.3, Proc Glmselect). Because the distribution of glucose was skewed, glucose concentration was log-transformed so that it could be reasonably fit by a linear model, and the values of biomarkers were standardized prior to analysis. During the selection process, all biomarkers and variables of subject characteristics were considered, and Schwartz Bayesian Criterion was evaluated for all models obtained by deleting a variable from the current model or adding a variable to this model. To avoid overfitting, a 10fold cross validation procedure was adopted to assess the performance, and the best model was determined based on the averaged predictive performance in test sets. For further analysis, this best model was applied to subgroups stratified by WHO body weight categories, with adjustment for additional covariates including age, BMI, and region (SAS 9.3, Proc Glmselect).

\section{Results}

3.1. Demographic Data. We enrolled 8,317 adolescents with a mean age of $10 \pm 0.7 \mathrm{y}$ in this study. The majority of adolescents were female $(61.2 \%)$. The average waist circumference was $68.7 \pm 19.4 \mathrm{~cm}$, and the average BMI of the population was $20.9 \pm 5.3 \mathrm{~kg} / \mathrm{m}^{2}$. The clinical characteristics based on body weight categories (underweight, normal weight, overweight, and obese) are shown in Table 1. About one-quarter (26.5\%) of the adolescents were obese.

3.2. Salivary Insulin Concentration by Body Weight Category. Given that insulin resistance and hyperinsulinemia are well-known features of the progression to a metabolically unhealthy obese phenotype in adolescents [17], we evaluated the salivary insulin concentrations from a cohort of 744 adolescents that were randomly selected from the larger 
study population across all body weight categories. It has been suggested that fasting blood insulin levels greater than $15 \mathrm{mU} / \mathrm{L}$ may be useful for evaluating insulin resistance [16]. Using the relationship we have previously established between blood and salivary levels of insulin [7], a correspondingly high insulin level in saliva would be $\geq 177 \mathrm{pg} / \mathrm{mL}$. With this value, we found that the prevalence of high salivary insulin concentration, indicating a state of insulin resistance and hyperinsulinemia, was increased in obese adolescents. None of the underweight adolescents, $4.3 \%$ of the normalweight adolescents, $8.3 \%$ of the overweight adolescents, and $25.7 \%$ of the obese adolescents had elevated salivary insulin concentrations $(p<0.0001)$.

\subsection{Salivary Glucose Concentration by Body Weight Category.} Continuing insulin resistance and hyperinsulinemia can lead to hyperglycemia [18], which is a hallmark of type 2 diabetes and the metabolic syndrome. Therefore, we analyzed glucose concentration in the saliva samples from the larger study population. As we have previously demonstrated, blood hyperglycemia $(\geq 100 \mathrm{mg} / \mathrm{dL})$ is approximated by the salivary glucose level of $\geq 1.13 \mathrm{mg} / \mathrm{dL}$ [10]. We were able to measure salivary glucose concentrations in $8,245(99.1 \%)$ of the saliva samples; 72 samples $(0.9 \%)$ were missing. The loss of samples was due to mechanical malfunction. The average salivary glucose concentration was $0.19 \pm 0.24 \mathrm{mg} / \mathrm{dL}$ (range: 0 $3.5 \mathrm{mg} / \mathrm{dL}$ ). Interestingly, there were no significant differences in the average salivary glucose concentration between body weight categories.

Among the subset of 744 adolescents randomly selected across all weight categories, overall $1.7 \%$ had salivary glucose levels suggestive of hyperglycemia ( $\geq 1.13 \mathrm{mg} / \mathrm{dL}$ ) (Figure 1). By weight category, salivary glucose levels were suggestive of hyperglycemia seen in none of the underweight adolescents, $1.1 \%$ of the normal-weight adolescents, $0.8 \%$ of the overweight adolescents, and 3.0\% of the obese adolescents $(p=0.89)$.

3.4. Salivary Glucose in Type 1 Diabetic Children. The validity of salivary diagnosis was suggested by a response to the question, "Do you have diabetes?" Thirty answered "yes" and also indicated that they were taking insulin. These children (presumably type 1 diabetics) had average salivary glucose of $0.27 \pm 0.39$. The other 8,138 children answered "no" with an average salivary glucose level of $0.19 \pm 0.24$. This difference was significant ( $p=0.013)$ suggesting that salivary glucose was higher in children diagnosed with type 1 diabetes.

\subsection{Relationship between Salivary Insulin and Glucose Con-} centrations and Obesity. We next compared the proportion of adolescents who had high salivary insulin concentration with the proportion of adolescents with high salivary glucose concentration among the 744 adolescents in the randomly selected cohort by body weight category (Figure 2). We found that while the prevalence of high salivary glucose and insulin concentrations both increased with obesity, they did not increase proportionally. About $25 \%$ of the obese adolescents were hyperinsulinemic but only $3.0 \%$ were hyperglycemic by

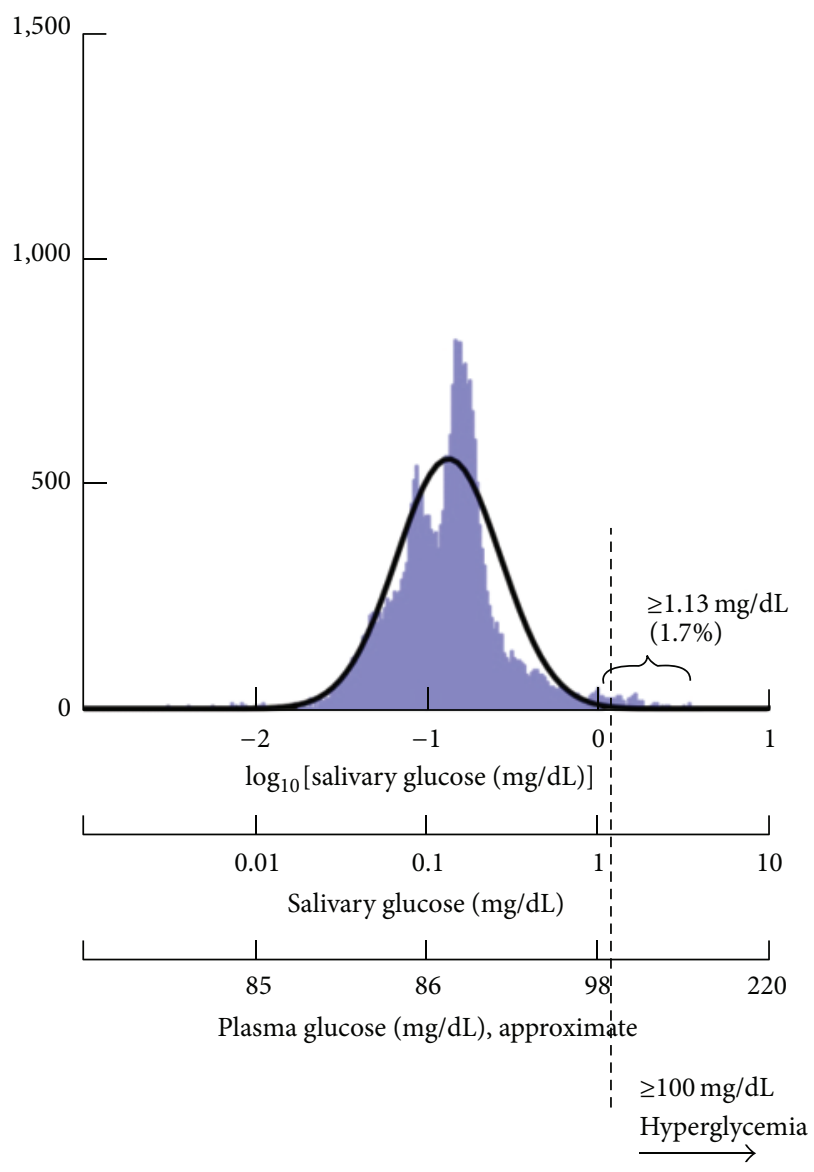

FIGURE 1: Semilogarithmic distribution of salivary glucose concentration values. Approximate values of the concentration of plasma glucose were computed from the equation [plasma $=13.5 \times$ saliva + 84.8] [10]. The salivary glucose concentration of $1.13 \mathrm{mg} / \mathrm{dL}$ was computed from the same equation and is approximately equivalent to a hyperglycemic plasma value of $100 \mathrm{mg} / \mathrm{dL}$.

our salivary analysis. In other words, in obese adolescents, the prevalence of high salivary glucose concentration increased only 3 -fold, while the prevalence of high salivary insulin concentration increased 6-fold, as compared to normalweight adolescents. A logistic binary regression analysis indicated that the odds ratio for elevated salivary glucose concentration in obese adolescents was 2.44 (95\% CI: 0.698.67) and for elevated salivary insulin concentration was 6.41 (95\% CI: 3.88-10.58). Therefore, in our cohort, obesity was significantly related to elevated salivary insulin concentration but not to elevated salivary glucose concentration.

\subsection{Predictors of Elevated Salivary Glucose Concentration} by Body Weight Category. Hyperglycemia is among the key factors indicating progression to a metabolically unhealthy obese phenotype in adolescents [19]. A simple analysis of correlation between salivary glucose and salivary biomarkers reveals that, of the 20 biomarkers measured, only insulin, VEGF, and IL-12p70 were significantly associated with each other (Table 2). To better understand the risk factors involved in the development of adolescent hyperglycemia, we built 
TABLE 2: Correlation coefficients $(r)$ with $p$ values between salivary glucose and salivary biomarkers sorted by ascending order of $p$.

\begin{tabular}{|c|c|c|c|c|c|}
\hline \multirow{2}{*}{ Biomarker } & \multicolumn{2}{|c|}{ Correlation coefficient } & \multirow{2}{*}{ Biomarker } & \multicolumn{2}{|c|}{ Correlation coefficient } \\
\hline & $r$ & $p$ & & $r$ & $p$ \\
\hline Insulin & 0.254 & $<0.0001$ & IL-10 & -0.066 & 0.255 \\
\hline VEGF & 0.162 & 0.005 & IL-8 & 0.063 & 0.275 \\
\hline IL-12p70 & -0.121 & 0.035 & MCP-1 & 0.058 & 0.312 \\
\hline MPO & -0.109 & 0.059 & IL-4 & -0.053 & 0.354 \\
\hline IL-13 & -0.092 & 0.108 & Leptin & 0.051 & 0.377 \\
\hline IL-17A & -0.089 & 0.121 & IL-1 $\beta$ & -0.043 & 0.452 \\
\hline Ghrelin & -0.087 & 0.132 & Adiponectin & -0.037 & 0.517 \\
\hline TNF- $\alpha$ & -0.084 & 0.145 & IL-6 & -0.035 & 0.548 \\
\hline Resistin & -0.078 & 0.176 & CRP & -0.033 & 0.561 \\
\hline IFN- $\gamma$ & -0.071 & 0.215 & MMP-9 & 0.011 & 0.845 \\
\hline
\end{tabular}

TABLE 3: Analysis of the association between salivary glucose, demographic factors, and biomarkers in a subset of 744 children stratified by body weight category. Values marked with an asterisk are statistically significant $(p<0.05)$.

\begin{tabular}{lcccccccc}
\hline & \multicolumn{2}{c}{ Underweight $(n=39)$} & \multicolumn{2}{c}{ Normal $(n=280)$} & \multicolumn{2}{c}{ Overweight $(n=121)$} & \multicolumn{2}{c}{ Obese $(n=304)$} \\
& Estimates $(\mathrm{SE})$ & $p$ value & Estimates $(\mathrm{SE})$ & $p$ value & Estimates (SE) & $p$ value & Estimates (SE) & $p$ value \\
\hline Age (per year) & $-0.26(0.18)$ & 0.16 & $-0.07(0.08)$ & 0.37 & $-0.07(0.13)$ & 0.52 & $0.02(0.07)$ & 0.84 \\
Sex (boys versus girls) & $0.23(0.29)$ & 0.43 & $-0.26(0.09)$ & $0.006^{*}$ & $-0.49(0.14)$ & $0.004^{*}$ & $-0.09(0.10)$ & 0.34 \\
BMI (per unit) & $0.16(0.17)$ & 0.35 & $-0.02(0.03)$ & 0.42 & $0.06(0.07)$ & 0.4 & $-0.01(0.01)$ & 0.54 \\
Insulin (per SD) & $0.52(0.73)$ & 0.48 & $0.16(0.08)$ & 0.06 & $0.25(0.11)$ & $0.02^{*}$ & $0.15(0.04)$ & $<0.0001^{*}$ \\
VEGFA (per SD) & $0.16(0.18)$ & 0.38 & $0.07(0.06)$ & 0.26 & $-0.08(0.12)$ & 0.53 & $0.10(0.04)$ & $0.01^{*}$ \\
IL-12p70 (per SD) & $-0.06(0.13)$ & 0.66 & $-0.07(0.05)$ & 0.13 & $-0.005(0.06)$ & 0.93 & $-0.14(0.05)$ & $0.01^{*}$ \\
Governorate & & $0.12^{\mathrm{a}}$ & & $<0.0001^{\mathrm{a}, *}$ & & $<0.0001^{\mathrm{a}, *}$ & $<0.0001^{\mathrm{a}, *}$ \\
\hline
\end{tabular}

${ }^{a} p$ value was for all governorates in a type 3 analysis.

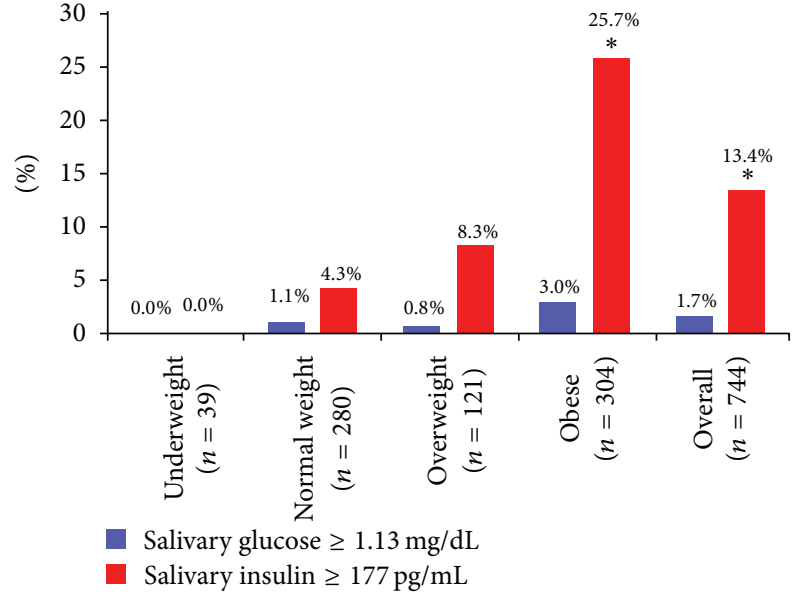

FIGURE 2: Percentage of the subset of 744 adolescents in each body weight category who had high salivary insulin and glucose concentrations. Only the values marked with an asterisk differed between high glucose and high insulin for each body weight category.

a predictive model for log-transformed salivary glucose levels that considered both clinical variables and salivary biomarker concentrations. The concentrations of 20 salivary biomarkers, including inflammatory markers and adipocytokines, were assayed in saliva samples from the cohort of 744 adolescents that were randomly selected from the larger study population across all body weight categories. Clinical variables from the larger study population were used for the benefit of a large sample size $(n=8,245)$. A data-driven stepwise selection procedure with 10 -fold cross validation yielded a parsimonious model containing sex, insulin, VEGF-A, and IL-12p70 as significant predictors of salivary glucose levels (Table 3). None of the other clinical variables nor any of the other 17 biomarkers in saliva that were tested qualified for this analysis.

Further analysis was conducted in subgroups by body weight category. In each group, a predictive model for salivary glucose concentration was built including the above selected predictors, as adjusted for age, BMI, and region. Among the clinical variables, the geographic region of Kuwait (governorate) was a significant factor in salivary glucose for all body weight classes except underweight. Participant sex was a significant predictor for salivary glucose concentration in the normal body weight group $(p=0.006)$ and the overweight group $(p=0.004)$.

Among the salivary biomarkers, none was a significant predictor for salivary glucose concentration in the underweight group. Salivary insulin concentration was a significant predictor for salivary glucose concentration in the overweight ( $p=0.02)$ group. In the obese group, the salivary concentrations of insulin, VEGF-A, and IL-12p70 were significant predictors for salivary glucose concentration, with insulin 
being the most robust ( $p<0.0001)$, followed by VEGFA $(p=0.01)$ and IL-12p70 $(p=0.01)$. Both insulin and VEGF-A were positively associated with salivary glucose concentration, while IL-12p70 showed an inverse association.

\section{Discussion}

Insulin resistance is a major factor in obesity-related, deleterious metabolic changes. Attenuated actions of insulin result in hyperglycemia, decreased protein synthesis, increased protein degradation, and increased susceptibility to infection [18]. Our current analysis of saliva samples from 8,245 Kuwaiti 10-year-old adolescents suggests that obesity in this population is significantly associated with high salivary insulin concentration but not with high salivary glucose concentration. Others have observed that hyperinsulinemia though often seen in children during puberty is not generally included as a risk factor for metabolic disease [20]. It has been suggested that fasting plasma insulin levels $>15 \mathrm{mU} / \mathrm{L}$ could be a reasonable clinical alternative for evaluating insulin resistance [16]. A very small percentage of adolescents in this study $(1.7 \%)$ had fasting salivary glucose levels that would be considered hyperglycemic $(\geq 1.13 \mathrm{mg} / \mathrm{dL}$ in saliva, equivalent to $\geq 100 \mathrm{mg} / \mathrm{dL}$ in plasma), and only $26.9 \%$ of those adolescents were obese. In contrast, a much larger percentage of the adolescents (13.4\%) had fasting hyperinsulinemia ( $\geq 177 \mathrm{pg} / \mathrm{mL}$ in saliva, equivalent to $\geq 15 \mathrm{mU} / \mathrm{L}$ in plasma), and $78 \%$ of those adolescents were obese. This observation supports the idea that a state of developing insulin resistance is present in these adolescents. The extraordinary differences in the fold increase of fasting salivary insulin concentration compared with fasting salivary glucose concentration in obese and overweight adolescents, however, suggest there may be factors outside the insulin-glucose control loop [21, 22] that accentuate insulin release. Exogenous stimulation of insulin release and resultant hypoglycemic episodes could be a reasonable mechanism by which obesity occurs in these adolescents.

In considering the progression from hyperinsulinemia to hyperglycemia, we conducted a salivary concentration analysis for 20 different biomarkers to determine their correlation with elevated salivary glucose concentration. VEGFA was one biomarker found to be positively correlated with salivary glucose concentration. VEGF-A is widely recognized as a signal protein that stimulates angiogenesis. Infusion of glucose alone has been shown to increase plasma VEGF levels [23], and elevated blood glucose in obese children has been associated with increased blood levels of VEGF-A [24]. Therefore, it is reasonable to assume that the process of developing increased body mass would be associated with an increased need for vascularization and, hence, increased levels of VEGF-A. It is also possible that the early association of increased VEGF-A levels with obesity and hyperglycemia could be related to the development of diabetic renal disease and/or retinopathy [25].

Interestingly, IL-12p70 levels in saliva were inversely correlated to salivary glucose levels. In addition to its ability to stimulate differentiation of native $\mathrm{T}$ cells into Th1 cells, IL-12p70 is also antiangiogenic [26]. Since the obese environment has both elevated levels of glucose and VEGFA, supporting angiogenesis in a growing body mass condition, inhibition of IL-12p70 could be part of this milieu. Indeed, it has been suggested that balance between proand antiangiogenic cytokines may be one of the factors that prevents diabetic kidney and retinal disease [27]. Further studies will be needed to elucidate the role of IL-12p70 in the development of hyperglycemia, insulin resistance, and obesity.

In summary, we observed that obesity in Kuwaiti adolescents was significantly related to high salivary insulin concentration but not to high salivary glucose concentration. For those adolescents who did have salivary glucose concentrations suggesting hyperglycemia, high salivary glucose concentrations were associated with increased levels of salivary insulin and VEGF-A and reduced salivary levels of IL-12p70. The current study expands our previous validation studies for glucose [10] and insulin [7] that indicated a significant correlation between plasma or serum concentrations and salivary concentrations of these molecules. Other investigators have presented data that indicate that salivary concentrations of VEGF-A [28] and IL-12p70 [29] are also significantly correlated ( $r=0.65,0.34$, resp.) with blood levels. Although we can only speculate at this stage, it seems that we are able to detect early changes in salivary biomarkers in 10 -year-old adolescents that could suggest the initiation of deleterious metabolic changes associated with obesity. Follow-up studies will focus on a wider selection of biomarkers to study in saliva to more precisely characterize these early changes. We will also expand our current observations to different populations of adolescents in order to increase clinical relevance.

Salivary concentrations of insulin and glucose can act as surrogates for their serum or plasma concentrations. We found that hyperinsulinemia may be more prominent than hyperglycemia in the early stages of obesity-related metabolic disease, and elevated insulin may be a dominant sign of metabolic disease. When hyperglycemia occurs in adolescents, it may be accompanied by a proangiogenic environment.

\section{Abbreviations \\ BMI: $\quad$ Body mass index \\ IFN: Interferon \\ IL: $\quad$ Interleukin \\ MMP-9: Matrix metalloproteinase-9 \\ RPM: Revolutions per minute \\ TNF- $\alpha$ : Tumor necrosis factor- $\alpha$ \\ WHO: World Health Organization \\ VEGF-A: Vascular endothelial growth factor-A.}

\section{Conflict of Interests}

The authors declare that there is no conflict of interests regarding the publication of this paper. 


\section{Authors' Contribution}

Mor-Li Hartman and J. Max Goodson are the guarantors of this work and, as such, had full access to all data in the study and take responsibility for the integrity of the data and the accuracy of the data analysis. Individuals conceiving and designing the study include J. Max Goodson, Francine Welty, and Kazem Behbehani. Individuals conducting clinical research included Hatice Hasturk, Jitendra Ariga, Sabiha AlMutawa, Ping Shi, Jawad Behbehani, and Roula Barake. Laboratory research personnel included Tina Yaskell and Danielle Stephens. Ethics approval and maintenance of review board status were by Maryann Cugini and Osama Alsmadi. Data analysis was conducted by Ping Shi and J. Max Goodson. All authors have participated in drafting and revising the paper and have approved the final version.

\section{Acknowledgments}

This research was funded by Dasman 10 from the Dasman Diabetes Institute, Dasman, Kuwait. The authors wish to recognize these individuals for helping to conduct the study in Kuwait from 2011 to 2012: Dr. Sarah Ahmed, Dr. Sumaiah, Dr. Yasmeen, Rabab Al Khajah, Massouma Saket, Laila Khaja, Laila Diab, Mariyam Sayer, Nadia, Mahmoud Bader, Amna Ahmed, Eman Adnan, and Shymaa Al Fadly. Saliva processing was done at Tissue Bank Core Facility, Dasman Diabetes Institute, Kuwait, under the supervision of Azadali Moorji, Head of TBCF. The authors also gratefully acknowledge the participation of dentists Rithna Shajahan, Safura Mohammed, Hemalatha Vasudevaraj, Enas Mahmoud Ismail, and Noha Syed Musthafa; nurses Wisam Hassan, Neda Saleh Hamad, Amal Kamel Ebrahim, Abeer Abdul Hameed, Amani Ata'allah, Flavia D. Ferrao, July K. Kunjumon, Reji Thomas, Wedad Al Shamary, Hanady Salem, Asma Al Said, AbdulHameed Said, Bashaer Eid Al Enezin, Jenny Mol Kurian, Laila Hamed Habib, Kholoud Kazem Jaber, Rawan Salem, Halima Malek Hussein, Marwa Ibraheem, Priya, Deslin, Suad Ahmed Al Deyab, Zakiya Abdul Mohsen, Rasha Mohammed, Kawthar Sa'd Jaber, Afaf Eid Tharesh, Nawal Abdullah, and Shaima Syed Mohammed; field coordinators Eman Sayed Khadmi and Fatma Al Fadhli; secretary Raseena Valamvayal; drivers A. K. Jamaluddeen, V. M. Kunhammed, K. K. Basheer, and O. T. Shaishad; and setup helpers P. Soopy and Shameer Porayil. They also acknowledge the assistance of New England Survey Systems of Brookline Massachusetts for aid in database preparation. The authors also thank Jillian Lokere, M.S., for editorial assistance in developing the paper. Ms. Lokere was paid by the Forsyth Institute, which derives its funds in part from a grant received from the Dasman Institute of Kuwait (a nonprofit institute) to study adolescent obesity in Kuwait.

\section{References}

[1] B. M. Popkin, "Does global obesity represent a global public health challenge?" The American Journal of Clinical Nutrition, vol. 93, no. 2, pp. 232-233, 2011.
[2] D. S. Freedman, L. K. Khan, M. K. Serdula, W. H. Dietz, S. R. Srinivasan, and G. S. Berenson, "The relation of childhood BMI to adult adiposity: the Bogalusa Heart study," Pediatrics, vol. 115, no. 1, pp. 22-27, 2005.

[3] N. Halfon, K. Larson, and W. Slusser, "Associations between obesity and comorbid mental health, developmental, and physical health conditions in a nationally representative sample of us children aged 10 to 17," Academic Pediatrics, vol. 13, no. 1, pp. 6-13, 2013.

[4] J. J. Reilly and J. Kelly, "Long-term impact of overweight and obesity in childhood and adolescence on morbidity and premature mortality in adulthood: systematic review," International Journal of Obesity, vol. 35, no. 7, pp. 891-898, 2011.

[5] J. M. Goodson and F. K. Welty, "Using salivary biomarkers to identify children at risk of Type 2 diabetes," Diabetes Management, vol. 4, no. 6, pp. 463-465, 2014.

[6] G. D. Foster, B. Linder, T. Baranowski et al., "A school-based intervention for diabetes risk reduction," The New England Journal of Medicine, vol. 363, no. 5, pp. 443-453, 2010.

[7] J. M. Goodson, A. Kantarci, M.-L. Hartman et al., "Metabolic disease risk in children by salivary biomarker analysis," PLoS ONE, vol. 9, no. 6, Article ID e98799, 2014.

[8] S. Karageorgi, O. Alsmadi, and K. Behbehani, "A review of adult obesity prevalence, trends, risk factors, and epidemiologic methods in Kuwait," Journal of Obesity, vol. 2013, Article ID 378650, 14 pages, 2013.

[9] International Diabetes Federation, IDF Diabetes Atlas, International Diabetes Federation, Brussels, Belgium, 5th edition, 2011.

[10] M.-L. Hartman, J. M. Goodson, R. Barake et al., "Salivary glucose concentration exhibits threshold kinetics in normalweight, overweight, and obese children," Diabetes, Metabolic Syndrome and Obesity: Targets and Therapy, vol. 8, pp. 9-15, 2015.

[11] R. K. Banerjee and A. G. Datta, "Salivary peroxidases," Molecular and Cellular Biochemistry, vol. 70, no. 1, pp. 21-29, 1986.

[12] A. Seidel, H. Parker, R. Turner et al., "Uric acid and thiocyanate as competing substrates of lactoperoxidase," The Journal of Biological Chemistry, vol. 289, no. 32, pp. 21937-21949, 2014.

[13] P. Piombino, A. Genovese, S. Esposito et al., "Saliva from obese individuals suppresses the release of aroma compounds from wine," PLoS ONE, vol. 9, no. 1, Article ID e85611, 2014.

[14] M. de Onis, A. W. Onyango, E. Borghi, A. Siyam, C. Nishida, and J. Siekmann, "Development of a WHO growth reference for school-aged children and adolescents," Bulletin of the World Health Organization, vol. 85, no. 9, pp. 660-667, 2007.

[15] P. Zimmet, G. K. M. M. Alberti, F. Kaufman et al., "The metabolic syndrome in children and adolescents-an IDF consensus report," Pediatric Diabetes, vol. 8, no. 5, pp. 299-306, 2007.

[16] C. L. Williams, L. L. Hayman, S. R. Daniels et al., "Cardiovascular health in childhood: a statement for health professionals from the Committee on Atherosclerosis, Hypertension, and Obesity in the Young (AHOY) of the Council on Cardiovascular Disease in the Young, American Heart Association," Circulation, vol. 106, no. 1, pp. 143-160, 2002.

[17] R. Vukovic, T. Milenkovic, K. Mitrovic et al., "Preserved insulin sensitivity predicts metabolically healthy obese phenotype in children and adolescents," European Journal of Pediatrics, vol. 174, no. 12, pp. 1649-1655, 2015.

[18] J. Boucher, A. Kleinridders, and C. Ronald Kahn, "Insulin receptor signaling in normal and insulin-resistant states," Cold Spring Harbor Perspectives in Biology, vol. 6, no. 1, Article ID a009191, 2014. 
[19] S. Blüher and P. Schwarz, "Metabolically healthy obesity from childhood to adulthood-does weight status alone matter?" Metabolism: Clinical and Experimental, vol. 63, no. 9, pp. 10841092, 2014.

[20] A. Jessup and J. S. Harrell, "The metabolic syndrome: look for it in children and adolescents, too!," Clinical Diabetes, vol. 23, no. 1, pp. 26-32, 2005.

[21] B. E. Corkey, "Banting lecture 2011: hyperinsulinemia: cause or consequence?” Diabetes, vol. 61, no. 1, pp. 4-13, 2012.

[22] B. E. Corkey, "Diabetes: have we got it all wrong? Insulin hypersecretion and food additives: cause of obesity and diabetes?" Diabetes Care, vol. 35, no. 12, pp. 2432-2437, 2012.

[23] M. Siervo, V. Tomatis, B. C. M. Stephan, M. Feelisch, and L. J. C. Bluck, "VEGF is indirectly associated with NO production and acutely increases in response to hyperglycaemia," European Journal of Clinical Investigation, vol. 42, no. 9, pp. 967-973, 2012.

[24] M. Siervo, D. Ruggiero, R. Sorice et al., "Body mass index is directly associated with biomarkers of angiogenesis and inflammation in children and adolescents," Nutrition, vol. 28, no. 3, pp. 262-266, 2012.

[25] M. Matsuda and R. A. DeFronzo, "Insulin sensitivity indices obtained from oral glucose tolerance testing: comparison with the euglycemic insulin clamp," Diabetes Care, vol. 22, no. 9, pp. 1462-1470, 1999.

[26] A. E. Yuzhalin and A. G. Kutikhin, "Interleukin-12: clinical usage and molecular markers of cancer susceptibility," Growth Factors, vol. 30, no. 3, pp. 176-191, 2012.

[27] K. Zorena, D. Raczyńska, and K. Raczyńska, "Biomarkers in diabetic retinopathy and the therapeutic implications," Mediators of Inflammation, vol. 2013, Article ID 193604, 11 pages, 2013.

[28] T. Upile, W. Jerjes, P. Kafas et al., "Salivary VEGF: a non-invasive angiogenic and lymphangiogenic proxy in head and neck cancer prognostication," International Archives of Medicine, vol. 2, no. 1, article 12, 2009.

[29] M. L. Byrne, N. M. O’Brien-Simpson, E. C. Reynolds et al., "Acute phase protein and cytokine levels in serum and saliva: a comparison of detectable levels and correlations in a depressed and healthy adolescent sample," Brain, Behavior, and Immunity, vol. 34, pp. 164-175, 2013. 


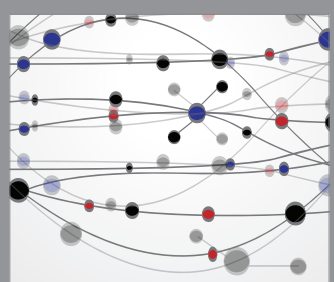

The Scientific World Journal
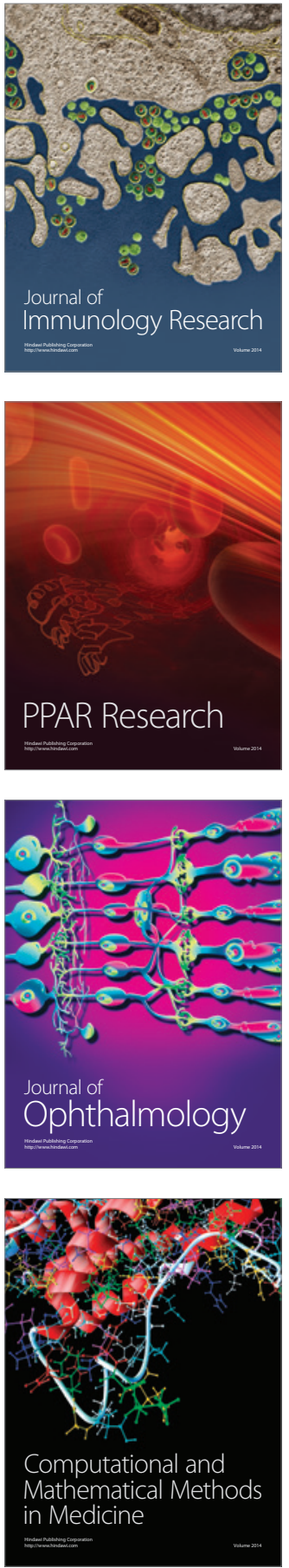

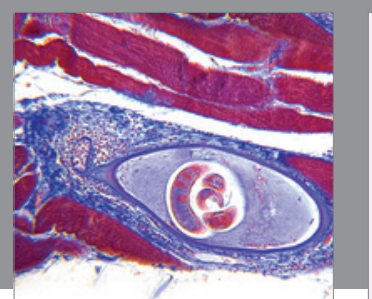

Gastroenterology Research and Practice

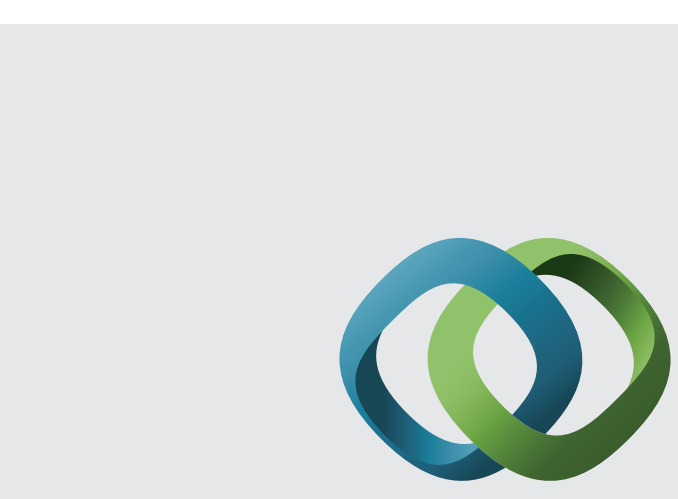

\section{Hindawi}

Submit your manuscripts at

http://www.hindawi.com
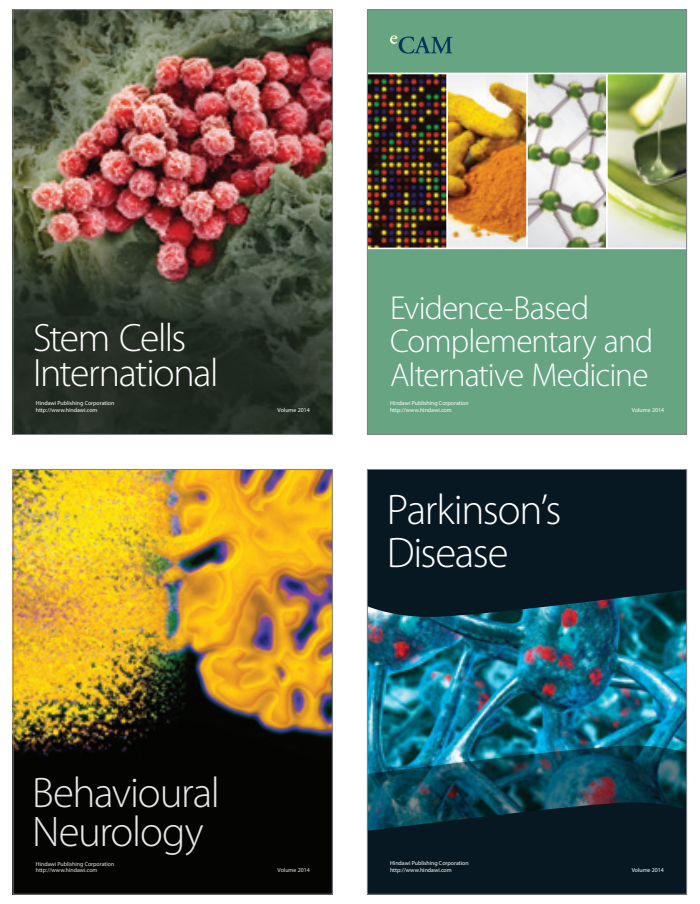
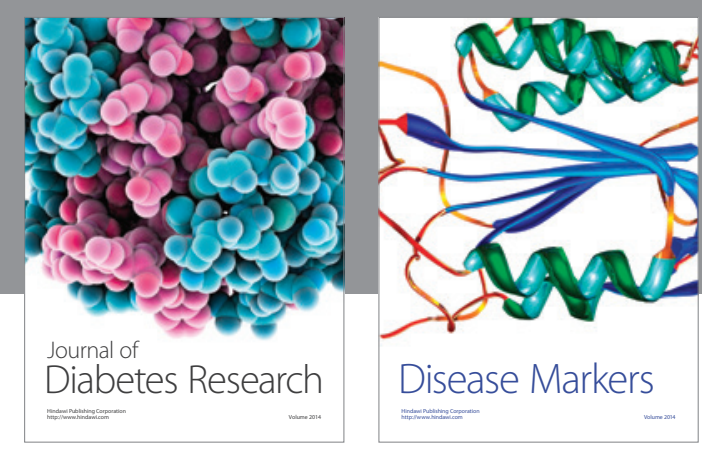

Disease Markers
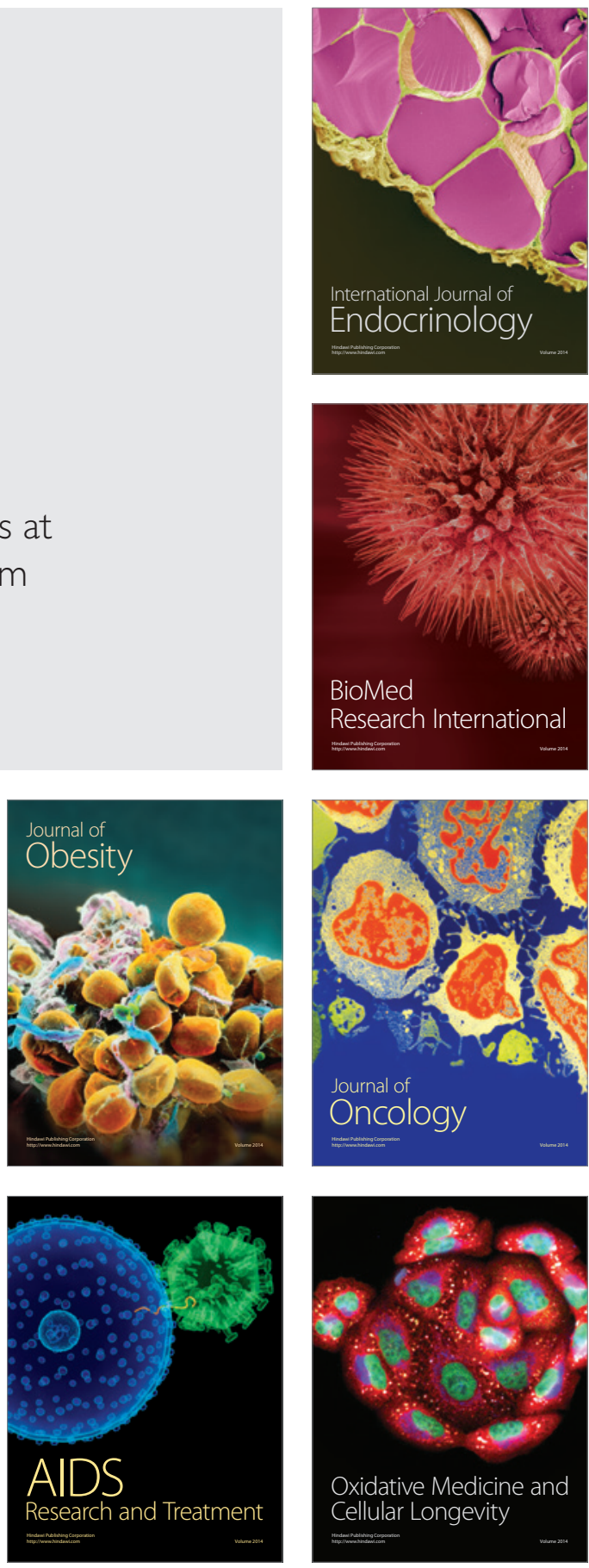